\title{
Can we test the influence of prosociality on high frequency heart rate variability? A double-blind sham-controlled approach.
}

\author{
Brice Beffara $^{1,2,3}$, Martial Mermillod ${ }^{1,2}$, Nicolas Vermeulen $^{3,4}$ \\ ${ }^{1}$ Univ. Grenoble Alpes, LPNC, F-38040, Grenoble, France \\ ${ }^{2}$ CNRS, LPNC UMR 5105, F-38040, Grenoble, France \\ ${ }^{3}$ IPSY, Université Catholique de Louvain, Louvain-la-Neuve, Belgium \\ ${ }^{4}$ Fund for Scientific Research (FRS-FNRS), Brussels, Belgium
}

\begin{abstract}
Author note
Correspondence concerning this article should be addressed to Brice Beffara, Office E250, Institut de Recherches en Sciences Psychologiques, IPSY - Place du Cardinal Mercier, 10 bte L3.05.01 B-1348

Louvain-la-Neuve, Belgium. E-mail: brice.beffara@univ-grenoble-alpes.fr

The polyvagal theory (Porges, 2007) proposes that physiological flexibility dependent on heartbrain interactions is associated with prosociality. So far, whether prosociality has a causal effect on physiological flexibility is unknown. Previous studies present mitigated results on this matter. In a randomized double-blind protocol, we used a generation of social closeness procedure against a standardized control condition in order to manipulate social affiliation as a prosocial interaction factor. High frequency heart rate variability (HF-HRV, indexing physiological flexibility), electromyographical activity of the corrugator supercilii (sensitive to the valence of the interaction) and self-reported measure of social closeness were monitored before, during, and after experimental manipulation. Cooperation was measured after the experimental manipulation as an index of behavioral prosociality. Data reveal no evidence toward and effect of the experimental manipulation on these measures. We discuss methodological aspects related to the experimental constraints observed in social psychophysiology. Implications for the experimental test of the polyvagal theory are approached within alternative theoretical frameworks.
\end{abstract}

Keywords: HF-HRV; autonomic flexibility; social closeness; double blind; prosociality;

cooperation

Word count: 6781

\section{Introduction}

Prosocial interactions are associated with positive health and ${ }^{18}$ well-being states (S. L. Brown \& Brown, 2015). More specif- ${ }^{19}$ ically, affiliate behaviors play an important part in coping ${ }^{20}$ with stressful events (Raposa, Laws, \& Ansell, 2015). Ac-21 cording to the polyvagal theory (Porges, 2007), heart-brain 22 interactions are a central mechanism in the interplay between 23 stress and prosocial behaviors. Particularly, efficient activity 24 of the myelinated vagus nerve connecting the heart and the 25 brain is proposed to foster capacities fully required when the 26 organism has to adapt to external demands and internal needs 27 such as during social interactions (Taborsky \& Oliveira, 2012). 28 This adaptability is referred as physiological or autonomic 29 flexibility (Brosschot \& Thayer, 1998; B. H. Friedman \& 30 Thayer, 1998; Thayer \& Lane, 2000) in reference to the abil- 31 ity of the organism to show dynamic variations in response 32 to the continuous variations of the environment. In the social domain, it has indeed been shown that physiological flexibility was associated with prosociality (Beffara, Bret, Vermeulen, \& Mermillod, 2016; J. G. Miller, Kahle, \& Hastings, 2015).

Even if important limitations have been suggested toward the polyvagal theory (Grossman \& Taylor, 2007; Taylor et al., 2014), it remains that more and more evidence corroborate the predicted link between the myelinated vagal functioning and affiliative social tendencies (Bornemann, Kok, Böckler, \& Singer, 2016; Kogan et al., 2014; Muhtadie, Koslov, Akinola, $\&$ Mendes, 2015). What remains unclear, however, is the direction of this association. Indeed, better heart-brain interactions could lead to improved social skills, or conversely, or even a third factor could link these two variables. Kok \& Fredrickson (2010) proposes that the association is actually bidirectional and that myelinated vagal activity and social ex- 
periences reciprocally influence each other in a dynamic loop. 86 This proposition is highly relevant since the development of 87 social skills likely depends on progressive learning processes 88 interacting with the evolution of cardiovascular regulation 89 (Brosschot, Verkuil, \& Thayer, 2016b, 2016a). However, the 90 method of Kok \& Fredrickson (2010) has been recently criti-91 cized, both on statistical and physiological aspects (Heathers, 92 Brown, Coyne, \& Friedman, 2015) but these criticisms have 93 been adequately answered (B. E. Kok \& Fredrickson, 2015). 94 The proposition remains important as it allows new hypothe- 95 ses formulations in the testing of the causal link between 96 the quality of heart-brain interactions and social function- 97 ing. However, to our knowledge, paradigms allowing to test 98 the causal direction in an experimental way have not been ${ }_{99}$ reported yet.

A recent meta-analysis (Shahrestani, Stewart, Quintana,101 Hickie, \& Guastella, 2015) on this matter concludes that ${ }^{102}$ positive social interactions do not increase myelinated vagal 103 functioning but negative interactions decrease it. One given ${ }^{104}$ explanation is that positive interactions could be beneficial ${ }_{105}$ after stressful events but not in a context already "favorable", which is in line with previous propositions (Raposa et al.,107 2015). The meta-analysis was performed on 14 studies in ${ }_{-108}$ cluding 17 tasks, among which 10 deal with negative social ${ }_{109}$ interactions (stressful), 3 with neutral interactions, and only $4_{110}$ with positive interactions. Looking more closely at the 4 posi $i_{-111}$ tive social tasks, we can observe that the manipulation of the ${ }_{112}$ valence of the social task in the positive direction leads hardly $y_{113}$ to conclude that a modulation of the affiliative functioning of ${ }_{114}$ the dyad actually happens.

Among the 4 studies dealing with positive interactions, the ${ }^{116}$ first reported in the meta-analysis has been carried-out by ${ }^{117}$ Butler, Wilhelm, \& Gross (2006). Their experimental ma-18 nipulation focused on an emotion regulation instructional ${ }^{119}$ set concerning a negative film. This instructional set was ${ }^{120}$ delivered for only one of the two members of the dyad. As ${ }^{121}$ a consequence, although the valence of the task was manip $\rightarrow 22$ ulated, affiliation was not technically central in their experimental design. The experimental manipulation of D'Antono,124 Moskowitz, Miners, \& Archambault (2005) was closer to $\mathrm{a}_{125}$ form of social closeness generation using agreeable versus ${ }_{126}$ quarrelsome role-play in dyads. However, as the prosocial ${ }_{127}$ nature and the effect (S. L. Brown et al., 2009) of agreeable ${ }_{128}$ role-play was not assessed, it is hard to determine whether ${ }_{129}$ myelinated vagal functioning was not influenced by provoked ${ }_{130}$ prosociality, or if prosociality was not actually successfully ${ }_{131}$ induced. On the contrary, Kathi J Kemper \& Shaltout (2011) ${ }_{132}$ seem to find an effect of prosociality on autonomic flexibility ${ }_{133}$ but the protocol includes non-verbal communication tech ${ }_{-34}$ niques which necessarily add confounding factors to the ma- -35 nipulation of more natural affiliative social behaviors. What ${ }_{136}$ is more, sample size was very small $(n=5)$ and the study was ${ }_{137}$ carried out in a healthy volunteer-clinician dyad, which $\operatorname{did}_{138}$ not allow applying the double blind during the experimental manipulation. Finally, because the sample size reported in Willemen, Goossens, Koot, \& Schuengel (2008) is larger, the increased autonomic flexibility observed after a positive social interactions is much more reliable. Nonetheless, the main methodological features of the experimental design do not allow to fully conclude to an effect specific to the prosocial interaction. Indeed, the study involves adolescent-parents interactions after a stressful event. The aim of the study was to determine the effect of the parent visit on stress recovery of the adolescent. As no control group was set up, there is still a possibility that the mere presence of another individual would have resulted in physiological modifications.

Collectively, this set of 4 studies gives important clues about the potential effect (or absence of effect) of prosocial interactions on autonomic flexibility. Despite all these efforts, we believed that the issue could be addressed by the mean of a complementary methodological design (S. L. Brown et al., 2009).

We used an experimental design based on the work of A. Aron, Melinat, Aron, Vallone, \& Bator (1997). This protocol enables to generate social closeness by the mean of guided discussions in dyads through short sentences such as questions or instructional sets. The content of these phrases promote the exchange of information between the two persons in the dyad. This exchange of information is expected to provoke reciprocal self-disclosure and engage the two persons in a prosocial interaction by sharing autobiographical elements about themselves. This has been shown to increase subsequent altruism (S. L. Brown et al., 2009). As a consequence, this design is particularly appropriate to test the polyvagal theory in the "social to physiology" direction. We also set up a combination of apparatus permitting to blind the experimenter to the condition of the participants (social closeness or a control condition also developed as a neutral "small talk" condition by A. Aron et al. (1997)).

As compared to several studies included in Shahrestani et al. (2015), we operationalized autonomic flexibility as the high frequency component of heart rate variability (HF-HRV, the variation in the cardiac beat to beat intervals, Heathers (2014)). HF-HRV is a reliable and noninvasive measurement of the dynamics of short-term heart-brain interactions (Thayer, Åhs, Fredrikson, Sollers, \& Wager, 2012). We also measured the electromyographical activity of the corrugator supercilii muscle (involved in frowning and emotional facial expressions of anger) as a secondary measure of autonomic activity. The corrugator supercilli activity is increased by negative and decreased by positive valence (J. T. Larsen, Norris, \& Cacioppo, 2003), which should be generated by our social closeness condition. Moreover, the corrugator supercilii is sensitive to threat (Costa, Bradley, \& Lang, 2015), which should be diminished by our social closeness condition. Both HF-HRV and corrugator supercilii activity should then be 
DOUBLE-BLIND PROTOCOL IN PROSOCIALITY INDUCTION

able to measure the effect of social closeness manipulations ${ }_{191}$ following the proposition of the polyvagal theory (Porges,, 92 2007).

193 Finally, our design also includes a task of cooperation after 194 the social closeness generation (or control) condition in order 195 to evaluate whether manipulating prosociality as affiliation ${ }_{196}$ and interpersonal positive contact can transfer to a behavioral 197 measure of prosociality. A dot detection task in dyads was 198 used, where the participant must try to press a key after a 199 signal, simultaneously with the other participant (X. Cheng, 200 $\mathrm{Li}, \& \mathrm{Hu}, 2015$; Cui, Bryant, \& Reiss, 2012). Cooperation is ${ }_{201}$ expected to increase response times in order to synchronize ${ }_{202}$ with the other participant while reducing the time between ${ }_{203}$ the response times of the two participants.

We hypothesized that, compared to a control condition, social ${ }_{205}$ closeness generation should increase HF-HRV, decrease the ${ }_{206}$ activity of the corrugator supercilii, and increase cooperation ${ }_{207}$ We also predict that this effect should be mainly observed in ${ }_{208}$ low baselines participants for HF-HRV and high baseline participants for EMG activity. Therefore, social closeness would ${ }^{209}$ benefit more to participants with lower autonomic flexibility ${ }^{210}$ and higher default stress response (Brosschot et al., 2016b, ${ }^{211}$ 2016a). Indeed, this hypothesis is based on a "deficit reme- ${ }^{212}$ diation" model according to which participants with deficits ${ }^{213}$ in a specific parameter will receive more benefits from the $e^{214}$ manipulation of this parameter (I. W. Miller et al., 2005, 2008). As a consequence lower/higher pre-manipulation ${ }^{216}$ HF-HRV/corrugator activation should predict higher level ${ }^{217}$ of progression after experimental manipulation. For instance, ${ }^{218}$ Davies, Niles, Pittig, Arch, \& Craske (2015) showed that ${ }^{219}$ cognitive behavioral therapy efficiency was higher for lower ${ }^{220}$ pre-manipulation HF-HRV participants, a mechanism we hy ${ }^{221}$ pothesize to apply also to our manipulation.

\section{Method}

Sample. Initial sample was composed of 104 healthy hu 226 man adults. Participants were recruited via advertisements ${ }^{227}$ (spread on facebook groups related to Louvain-la-Neuve, Bel 228 gium, where the experiment took place). Participants were 229 from the general population and were French speaking. They 230 provided written informed consent before the participation? ${ }^{231}$ The study was reviewed and approved by the ethics commis ${ }^{232}$ sion of the Psychological Sciences Institute of the Catholic ${ }^{233}$ University of Louvain, Belgium (reference number 15-37) 234 To be eligible, participants had to be aged between 18 and $60_{235}$ years, with a normal or normal-to-corrected vision, explicitly ${ }_{236}$ reported an absence of psychiatric, neurological, hormonal, or $_{237}$ cardiovascular disease, and with no medical treatment (with 238 the exception of contraception). Smoking, energizing drinks 239 (e.g. coffee, tea, etc...) and psychotropic substances (e.g. al-240 cohol, cannabis, etc...) were prohibited to each participant 241 the day of the experiment. They had also to avoid eating ${ }_{242}$ or drinking (water was allowed) the 2 hours preceding the 243 experiment in order to limit the influence of digestion on autonomic functioning (Short term HRV measurement can be biased by the digestion of food since viscera are innervated by the autonomic nervous system, (Heathers, 2014; Iorfino, Alvares, Guastella, \& Quintana, 2016; Quintana \& Heathers, 2014)) but they had to eat in the morning (more than 2 hours before the experiment) in order to avoid fasting states. The participants received experimental 15 euros at the beginning and 20 euros at the end of the period the recruitment in order to complete our sample.

Sample size. We planned one hundred and sixty participants to take part in the study in order to work with a similar sample size as compared to S. L. Brown et al. (2009). Their sample size was adequate to observe an effect of an experimental generation of social closeness on progesterone compared to a neutral control task, with an effect size of $R^{2} \sim .63$. Unfortunately, even with an increase of the compensation, we could not reach this sample size.

Procedure. After completing the inclusion survey online, participants suitable for participation were automatically redirected toward another survey in order to give their available dates for an appointment (others were thanked and informed that they did not fit with the criterion). A homemade R-script was built in order to randomly select dyads among all participants available at each slot. The appointment date and time was determined and communicated to the participants roughly $72 \mathrm{~h}$ before the actual slot. The experiment took place in a quiet and dimmed room. All participants were tested between $0900 \mathrm{~h}$ and $1300 \mathrm{~h}$. Participants were asked to go empty their bladder before starting the experiment. After a global description of the experiment, they were taught how to install the

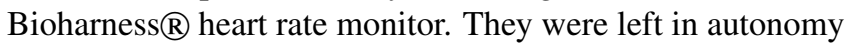
in an isolated room for the installation of the heart rate monitor. Then, they seated in a chair, the experimenter checked the signal and the installation of EMG electrodes began. The three electrodes (two on the corrugator supercilii and on the top of the forehead) were attached and the signal was checked. Classical piano music (Ballade No.4 in F minor, Op. 52 by Frederic Chopin, interpreted by Franck Levy https://musopen. org/fr/music/769/frederic-chopin/ballade-no-4-op-52/) was played during the installation. We added this feature in order to compensate for the potential stressful effects of electrodes' installation. The experiment started when the quality of the signals were correct.

First participants had to perform facial actions in order to get a baseline of the volitional contraction of the corrugator supercilli. They had a succession of $2 * 10=20$ instructional sets randomly displayed on their computer screen: "Frown then relax", "Swallow", "Wrinkle your eyes then relax", "Clench the jaws then relax", "Close then open your eyes", "Close then open your mouth", "Raise the corner of your lips then relax", "Raise your eyebrows then relax", "Wrinkle your nose then relax", "Lower the corner of your lips then relax". The 
"Frown then relax" instructional set was used to compute 297 maximum possible signal level and other instructions had a 298 distraction role in order to avoid too much focusing on the 299 frowning action during the experiment. Instructions were ${ }_{300}$ displayed for 3 seconds on the screen and followed by a $3_{301}$ second new instruction to relax the face.

302 In a second step, participants were asked to answer some ques-303 tions about their relationship with the their partner (i.e. the ${ }^{304}$ other participant of the dyad, (A. Aron \& Fraley, 1999; A305 Aron et al., 1997)). First, they were asked if they knew their ${ }^{306}$ partner on a Likert scale from 1 = "Not at all" to 7 = "Perfectly307 well". Second, they were asked how close they felt to their 308 partner on a Likert scale from $1=$ "Not at all", from $7=309$ "Enormously".

310 During the 5 following minutes, participants watched short ${ }^{311}$ neutral samples of films selected and evaluated by Hewig et ${ }^{312}$ al. (2005) ("Hannah and her Sisters" and "All the President's ${ }^{313}$ Men") and Schaefer, Nils, Sanchez, \& Philippot (2010) ("Blue ("14 $^{314}$ [1]", "Blue [2]", "Blue [3]" and "The lover"). Videos were ${ }^{315}$ displayed without audio. These first 5 minutes aimed to allow ${ }^{316}$ participants to shift in a resting state. ECG data for HRV base- ${ }^{317}$ line computation was recorded for the 5 following minutes ${ }^{318}$ while participants listened to the first 5 minutes of a neutral ${ }^{319}$ audio documentary designed for laboratory studies (Bertels, ${ }^{320}$ Deliens, Peigneux, \& Destrebecqz, 2014). Neutral videos and ${ }^{321}$ audio documentary were used in order to standardize $\mathrm{ECG}_{322}$ recordings (Piferi, Kline, Younger, \& Lawler, 2000). After resting-state recording, participants were put in a dis-324 cussion situation for a minimum of 10 minutes. The protocol 325 is detailed below. Another resting-state recording was per-326 formed after the discussion for 5 minutes while participants 327 listened to the last 5 minutes of the neutral audio documentaryз28 (Bertels et al., 2014). Then participants performed the cooper- -29 ation task detailed below. The last step of the experiment in ${ }^{330}$ laboratory was again a question about how close participants 331 felt to their partner on a Likert scale from $1=$ "Not at all",, 32 from 7 = "Enormously". EMG electrodes were detached ${ }_{3 з}$ (classical music was played), the ECG belt uninstalled, and aз34 debriefing was proposed before compensating the participants 335 Control survey was completed at home, online, on Qualtrics,336 thanks to an identifier given to the participants. ECG dataз37 was recorded during spontaneous breathing (Denver, Reed, $\&_{338}$ Porges, 2007; Kobayashi, 2009; Kowalewski \& Urban, 2004; P. D. Larsen, Tzeng, Sin, \& Galletly, 2010; Muhtadie et al. ${ }_{340}$ 2015; Pinna et al., 2007). The experimenter was available at ${ }_{34}$ any time during the experiment but stayed in another room.

Generation of social closeness. Dyads were randomly and $\mathrm{a}_{343}$ automatically assigned to the closeness generation condition ${ }_{344}$ or control condition. Participants were blind to their condi-345 tion, such as the experimenter. Both conditions were guided ${ }_{346}$ discussions where participants took turn asking a question to 347 their partner -and the partner had to answer the question- or $_{348}$ following a small instruction in order to engage in conversa-349 tion. The phrases used to guide the conversation were taken from A. Aron et al. (1997), translated in French by external translators (3 pairs of translation and back-translation) and reviewed, adapted, and selected by us. The minimum time for discussion was 10 minutes, but could last a bit more depending on the duration of the last item (phrase). Thirty-six items were available for the discussion which was largely enough to fill 10 minutes, even if participants move quickly from one item to another. Items were displayed sequentially and the participants chose to move to the following item. Items were used for each participant (participant 1 asks participant 2 and vice versa). In the social closeness condition, phrases are designed to foster, as described by A. Aron et al. (1997) "sustained, escalating, reciprocal, personalistic self-disclosure" (A. Aron et al., 1997, p. 364) such as "Given the choice of anyone in the world, whom would you want as a dinner guest?", "When did you last sing to yourself? To someone else?", or "Is there something that you've dreamed of doing for a long time? Why haven't you done it?". In the control condition phrases are more neutral and less likely to engage this kind of process, such as "When was the last time you walked for more than an hour? Describe where you went and what you saw.", "If you could invent a new flavor of ice cream, what would it be?", "Where are you from? Name all of the places you've lived.”.

During all the experiment, the participants were seated opposite to each other but separated by a panel such that they could not see each other. Each participant had his/her own screen, and the two screens were connected to the same computer. A webcam was attached to each screen and was automatically activated at the beginning of the discussion phase and shut down at the end of the same phase. The video from the webcam was displayed in real time on the screen of the other participant. As a result, participants could see each other only during the discussion phase by the mean of the webcam. We programmed, using the OpenCV library for Python, the automatic management of the web-cams and their coordination. The script was integrated in the Psychopy 1.8 script in order to match the progress of the experiment. Web-cams were calibrated at the beginning of each testing in order to center the image on the face of the participant.

\#\#\#\# Cooperation We used the cooperation task described in Cui et al. (2012) and X. Cheng et al. (2015) in order to evaluate behavioral prosociality after experimental manipulation. This task includes a cooperation task, competition task and a neutral task. The competition and neutral tasks serve as control tasks. Each trial begins with a hollow gray circle at the center of the screen of each participant that stays visible for a random interval between 0.6 and $1.5 \mathrm{~s}$. Subsequently, a green cue signals the participants to press a response key (left arrow for one and right arrow for the other. A green sticker was attached to the keys in order to make them salient on the keyboard). During a training phase of 5 trials, participants 
had to be relatively constant in their response times. Their 403 response time was displayed as a feedback after the first trial ${ }_{, 04}$ and then the feedback was "+ 1 point" if they succeeded in 405 being constant or "-1 point" if they failed for the following ${ }_{406}$ trial. A response time was determined as constant compared 407 to the previous trial if the difference between the two trials 408 was inferior to a threshold $\mathrm{T}=(\mathrm{RT} 1+\mathrm{RT} 2) / 10$ (adapted from ${ }_{409}$ Cui et al. (2012)). The aim of the participant was to get $\mathrm{a}_{410}$ maximum number of points for each phase. The score was $_{411}$ reset to 0 at the end of each phase.

The first phase of interest was the phase of cooperation com $_{413}$ posed of 20 trials. Participants had to coordinate (without ${ }_{414}$ talking to each other) in order to press the key simultaneously ${ }_{415}$ A trial was successful if the difference of response times ${ }_{416}$ between the two players was inferior to $\mathrm{T}=(\mathrm{RT} 1+\mathrm{RT} 2) / 1_{\mathrm{A}_{17}}$ Again, a feedback " +1 point" was displayed if they succeeded ${ }_{41}$ in cooperating or "-1 point" if they failed.

The second phase was the competition phase where the aim $_{420}$ was to press the key faster than the other player. The fastest ${ }_{421}$ player won 1 point and the slowest lost one. The third and a22 $_{2}$ last phase was played alone with the same instructions as the ${ }_{423}$ training phase detailed above.

424 The points were uniquely related to the task and had no other ${ }_{425}$ consequences on the experiment. Each negative feedback ${ }_{426}$ ("-1 point") was associated with the display of the differ $_{-427}$ ence between response times. In the "competition" phase ${ }_{428}$ the difference between response times was always displayed ${ }_{429}$ alongside the points. The task was coded in Python.

\section{Physiological measurement.}

Electrocardiography. The electrocardiogram (ECG) data ${ }^{432}$ was recorded with a Zephyr Bioharness ${ }^{\mathrm{TM}} 3.0$ (Zephyr, 2014). ${ }^{433}$ The Bioharness ${ }^{\mathrm{TM}}$ is a class II medical device presenting a ${ }^{434}$ very good precision of measurement for ECG recording in ${ }^{435}$ low physical activity conditions (Johnstone, Ford, Hughes, ${ }^{436}$ Watson, \& Garrett, 2012a, 2012b; Johnstone et al., 2012). It ${ }^{437}$ has been used for ECG measurements in both healthy and ${ }^{438}$ clinical populations, presenting a very high-to-perfect correla-739 tion with classical hospital or laboratory devices (Brooks et 440 al., 2013; Yoon, Shah, Arnoudse, \& De La Garza, 2014). The ${ }_{441}$ Bioharness ${ }^{\mathrm{TM}}$ both provides comfort for the participant and $\mathrm{al}_{-442}$ lows reliable HRV extraction for the researcher (Lumma, Kok ${ }_{, 43}$ $\&$ Singer, 2015). The chest strap's sensor measures electrical ${ }_{444}$ activity corresponding to the classical V4 lead measurement $t_{45}$ (5th intercostal space at the midclavicular line) through con $_{-446}$ ductive Lycra fabric. A single-ended ECG circuit detects $\mathrm{QRS}_{447}$ complexes and incorporates electrostatic discharge protection ${ }_{448}$ both active and passive filtering and an analog-to-digital con-449 verter. Interbeat intervals are derived by Proprietary digital ${ }_{450}$ filtering and signal processed with a microcontroller circuit ${ }_{451}$ The ECG sensor sampling frequency is $250 \mathrm{~Hz}$ and the res-452 olution $0.13405 \mathrm{mV}$, ranging from 0 to $0.05 \mathrm{~V}$ (Villarejo, 453 Zapirain, \& Zorrilla, 2013). After a slight moistening of the 2454 ECG sensors, the chest-strap was positioned directly on the 455 skin, at the level of the inframammary fold, under the lower border of the pectoralis major muscle. The recording module communicated with an Android $\circledast$ OS smartphone by Bluetooth $\AA$. The application used to acquire the signal emitted by the Bioharness ${ }^{\mathrm{TM}}$ was developed, tested, and validated by Cânovas, Domingues, \& Sanches (2011). The Android® OS device used to record the signal was an LG-P990 smartphone (Android® version 4.1.2.).

Electromyography. EMG was measured with three $4 \mathrm{~mm}$ $\mathrm{Ag} / \mathrm{AgCl}$ electrodes: two electrodes were attached at the level of left brow (central part, just above the brow) and one ground sensor was placed upon the participant's top left forehead (Fridlund \& Cacioppo, 1986). Sampling frequency was set at $2000 \mathrm{~Hz}$.

Control for confounding factors. To control for confounding variables likely to be linked to HRV, participants completed questionnaires detailing life habits, demographic data and emotional traits (Quintana, Guastella, Outhred, Hickie, \& Kemp, 2012). Physical activity was assessed with the International Physical Activity Questionnaire (IPAQ,Craig et al. (2003)), composed of 9 items that calculate an index reflecting the energy cost of physical activities (Metabolic Equivalent Task score, MET). The IPAQ has been validated in French (Briancon et al., 2010; Hagströmer, Oja, \& Sjöström, 2006) and widely used in French surveys (Salanave et al., 2012). Participants also completed the Depression Anxiety and Stress scales (DASS-21;(P. F. Lovibond \& Lovibond, 1995)). The DASS-21 is a 21 -item questionnaire, validated in French (Ramasawmy \& Gilles, 2012), and composed of three subscales evaluating depression, anxiety and stress traits. We also recorded the size, weight, age and sex of the participants and their daily cigarette consumption. Participants answered final surveys at home via an online survey built with Qualtrics in order to reduce the time spent in the laboratory and to allow all the dyads to be tested between $0900 \mathrm{~h}$ and $1300 \mathrm{~h}$.

\section{Physiological signal processing.}

Electrocardiography. R-R interval data was extracted from the Android $\AA$ device and imported into RHRV for Ubuntu (Rodríguez-Liñares et al., 2011). Signal was visually inspected for artifact (Prinsloo et al., 2011; Quintana et al., 2012; Wells, Outhred, Heathers, Quintana, \& Kemp, 2012). Ectopic beats were discarded (Kathi J. Kemper, Hamilton, \& Atkinson, 2007) for participants presenting a corrupted RR interval series (Beats per minute (bpms) shorter/longer than $25 / 180$ and/or bigger/smaller than $13 \%$ compared to the 50 last bpms). RR series were interpolated by piecewise cubic spline to obtain equal sampling intervals and regular spectrum estimations. A sampling rate of $4 \mathrm{~Hz}$ was used. We then extracted the frequency component of HRV from RR interval data. The LF $(0.04-0.15 \mathrm{~Hz})$ and HF $(0.15-0.4 \mathrm{~Hz})$ components were extracted using an east asymmetric Daubechies wavelets with a length of 8 samples. Maximum error allowed was set as 0.01 (García, Otero, Vila, \& Márquez, 2013). 


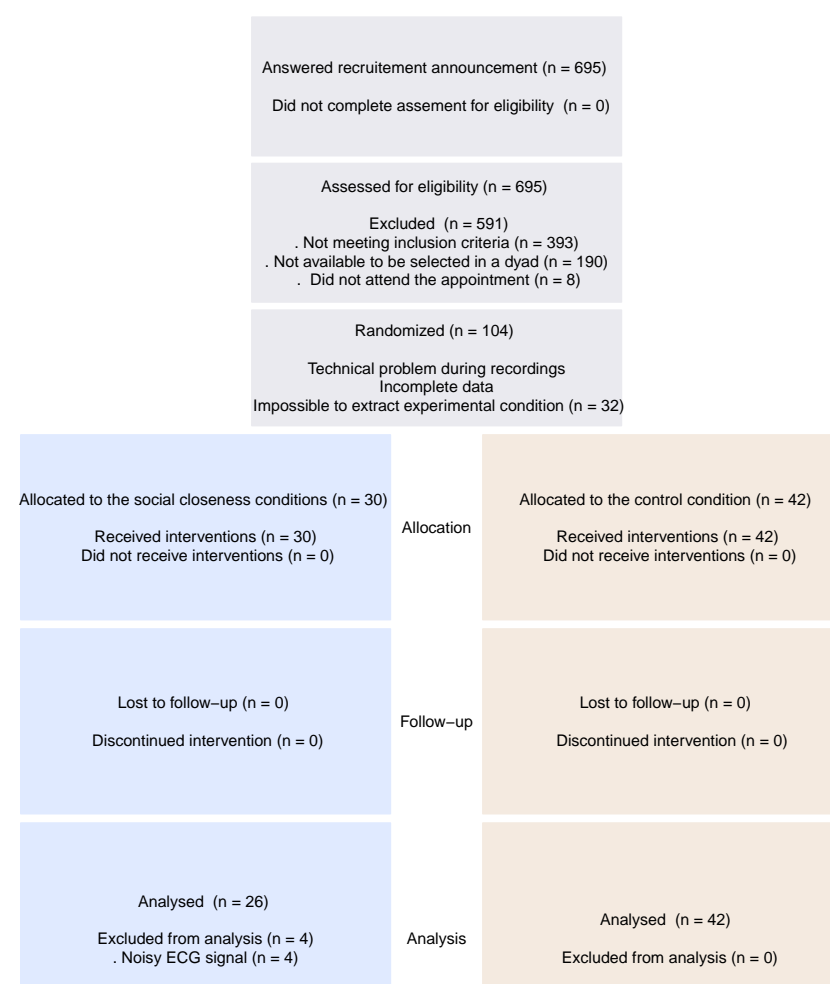

Figure 1. Flow chart of participants

Electromyography. Signals were re-sampled at $1000 \mathrm{~Hz}$ amplified, filtered through a $30-250 \mathrm{~Hz}$ band pass and $60^{509}$ $\mathrm{Hz}$ notch, digitized, re-filtered, rectified, and then integrated over $20 \mathrm{~ms}$ (Bershad, Seiden, \& Wit, 2016) online using EMG100C amplifiers, an MP150 data Acquisition System, and Acqknowledge software from Biopac Systems (Goleta ${ }_{5,4}$ CA, USA). Maximum acceptable amplitude of the signal was computed as the maximum signal value during the volitional ${ }_{516}^{515}$ contraction of the corrugator supercilii (frowning). All values superior to this threshold were reduced as the threshold value. EMG files presented a median [interquartile range] of $0.00[0$ $-0.01] \%$ of ectopic EMG values with a maximum value of $2.13 \%$.

\section{Data analysis.}

Data preparation. Excluding technical problem record-523 ings^[Numerous recording problems happened notably due to524 frequent freezing of Psychopy when managing the synchro-525 nization of the two web-cams. Moreover, a lot of participants526 lost their identifier and did not complete the control ques-527 tionnaires, we therefore excluded these data for final analysis 528 for all participants. Here we present the results concerning a529 restricted part of our sample where all data (excepted demo-530 graphic and self-reported at home) are available, a total of $72_{531}$ participants were available for data analyses (Figure 1). 4 par-532 ticipants were excluded from the sample before data analysis sз3 $_{3}$ because of a noisy ECG signal. Analysis on physiological signals were performed on data averaged on 5 minutes for each experimental step $(\mathrm{t} 1=$ resting baseline, $\mathrm{t} 2=$ first part of the discussion, $\mathrm{t} 3=$ second part of the discussion, $\mathrm{t} 4=$ resting after discussion). As a result, 4 measurement points of 5 minutes were available for 2 physiological measurements (HF$\mathrm{HRV}$ and $\mathrm{EMG}_{\text {corrugatorsupercilii }}$. All participants included in final analysis presented a median [interquartile range] of 0.03 $[0-0.16] \%$ of ectopic beats values with a maximum value of $8.17 \%$. We computed the HF power of HF-HRV baseline and then calculated as the natural logarithm of the HF power in order to correct the right-skewed distribution (Kogan et al., 2014; Pinna et al., 2007). In order to correct for the positive skew of EMG data, data were square-root transformed (J. T. Larsen et al., 2003) but normality could not be reached for each time step. Behavioral data in the cooperation task (measured after $\mathrm{t} 4$ ) were analyzed as raw reaction times and difference of reaction times between participants of the dyad as a function of the experimental group.

Model comparison. Statistical analysis were conducted using RStudio $($, version 1.0.8 for Linux (R Core Development Team, 2015) and are reported with the knitr (Xie, 2013), papaja (Aust \& Barth, 2015) and rmarkdown (Allaire et al., 2016) packages. The aim of data analysis was to detect whether experimental social closeness generation (compared to the control condition) influenced physiological and behavioral variables across time and whether this effect was dependent on the baseline level of participants. We compared alternative models (for alternative hypothesis = "H1") with experimental group (called "G": Closeness vs. Control) and models with experimental group and resting baseline (called "R" = t 1$)$ as an independent variable to a model containing only the intercept (Null hypothesis = "H0" model) with signals at each time step and channel as dependent variables. Behavioral data measured during the cooperation task at the end of the experiment (after t4) were analyzed as a function of the experimental group and task type (competition session, alone situation session, and cooperation session, respectively coded as $-1,0,1)$.

We analyzed our data by the fit linear mixed-effects models function (lmer), computed using the package "Ime4" [Bates et al. (2014);Bates2015] for behavioral data and linear models function ( $\mathrm{lm}$ ) for physiological data (Chambers, 1992). All residuals of the models were not normally distributed but data transformation did not allow getting normal distribution. Model selection was completed using AICc (corrected Akaike information criterion) and Evidence Ratios - $E R_{i^{-}}$(K. P. Burnham \& Anderson, 2004; Kenneth P. Burnham, Anderson, \& Huyvaert, 2011; Hegyi \& Garamszegi, 2011; Symonds \& Moussalli, 2011). In this perspective, all the hypotheses are considered equally, meaning that the status of $\mathrm{H} 0$ (absence of effect) is the same as compared to H1 (effect), all models can be compared together. AIC provides a relative measure of 


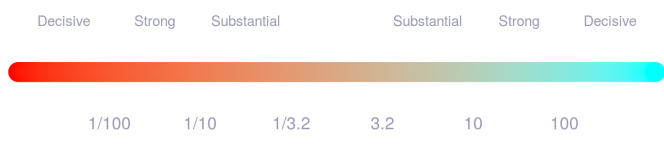

Evidence ratio

Figure 2. Interpretation of evidence ratios relatively to $\mathrm{H} \mathrm{O}_{579}$ and $\mathrm{H} 1$.

goodness-of-fit but also of parsimony by sanctioning models ${ }^{583}$ for their numbers of parameters. AICc is more severe on this 584 last point than $\operatorname{AIC}\left(A I C c=A I C+\frac{2 K(K+1)}{n-K-1}\right.$ where $K$ is the ${ }_{586}^{585}$ number of parameters and $n$ the sample size.). We computed ${ }_{587}$ the difference between best (lower value of AICc) and other ${ }_{588}$ AICcs with $\Delta_{A I C c}=A I C c_{i}-A I C c_{\min }$ thanks to the piecewis- ${ }_{589}$ eSEM package (Lefcheck, 2016). The weight of a model is ${ }_{590}$ then expressed as $w_{i}=\frac{e^{\frac{1}{2} \text { Delta }_{A I C c_{i}}}}{\sum_{r}^{R} e^{\frac{1}{2} \Delta_{A I C c_{r}}}}$. From there, we can com- ${ }^{591}$ pute the Evidence Ratio between the alternative model and ${ }_{593}$ the intercept model: $E R_{\text {alt }}=\frac{w_{\text {alt }}}{w_{\text {int }}}$. For each physiological and ${ }^{594}$ behavioral measurement, we were able to compare the effect of the group and the interaction effect between the intervention group ("G") and the baseline ("R" = t1 for physiological measurement, only for physiological data) of the participants with the "H0 model" including only the intercept. Baselines were set as continuous factors (for physiological data only) and experimental groups (group: Closeness vs. Control) were coded as +0.5 and -0.5 respectively. If the alternative model (for $\mathrm{H} 1$ ) is more parsimonious than the intercept model (for H0) then substantial $(3.2<E R<10)$, strong $(10<E R<100)$ or even decisive $(100<E R)$ evidence should be observed (Kass \& Raftery, 1995; Snipes \& Taylor, 2014). On the contrary, substantial $(1 / 3.2<E R<1 / 10)$, strong $(1 / 10<E R<1 / 100)$ or even decisive $(1 / 100<E R)$ evidence toward the intercept model would allow concluding that the intercept model is more parsimonious. An $1 / 3.2<E R<3.2$ do not allow to draw conclusions and indicates that the data does not provide significant evidence toward one model or the other (Figure 2).

\section{Results}

We first analyzed self-reported measures obtained on Likert scales (7 points), measuring how much participants knew the other participant of the dyad before the experiment, and how much they felt close to him/her before and after the experiment. Participants knew each other similarly and also felt equally close before the experiment in each group (Table 1). Indeed, evidence ratios $(<3.2)$ do not permit to conclude to a difference between groups. The scores between groups were neither different after the experiment, hwever there was strong evidence toward an increase of social closeness in both groups (ER > 100 toward the intercept model compared to 0). Looking at physiological data (Table 2), we did not observe substantial differences between the two conditions (Table 3). Contrary to our hypothesis, there was no evidence toward an interaction between the experimental condition and the baseline. Indeed, all evidence ratios were inferior to 3.2 and, contrary to our hypothesis, do not allow to conclude neither to an effect of social closeness manipulation nor to an interaction with initial physiological levels.

The same result appeared concerning behavioral data ${ }^{1}$. Contrary to our hypothesis, there was no evidence toward an effect of group and task type (cooperation, competition, and the single condition) on reaction times, nor on differences of reaction times between participants of a same dyad. Only the task type had a strong effect on reaction times following a linear relationship (competition, alone situation, and cooperation respectively coded as -1, 0, 1, Tables 4) and 5. Again, contrary to our hypothesis, participants in the social closeness condition did not differ from participants in the control condition in terms of reaction times in the dot detection task, either for cooperative, competitive, or alone situation. Experimental manipulation did not modify behavioral response synchronization between participants of a same dyad.

\footnotetext{
${ }^{1}$ No transformation allowed to get normality distribution on reaction times
} 
bioRxiv preprint doi: https://doi.org/10.1101/078006; this version posted September 28, 2016. The copyright holder for this preprint (which was not certified by peer review) is the author/funder, who has granted bioRxiv a license to display the preprint in perpetuity. It is made available under aCC-BY-NC-ND 4.0 International license.

8

BRICE BEFFARA ${ }^{1,2,3}$, MARTIAL MERMILLOD ${ }^{1,2}$, NICOLAS VERMEULEN ${ }^{3,4}$

Table 1

Effect of experimental group on self-reported measures compared to the intercept model. $E R=$ evidence ratio, SC $=$ Social closeness group, $C T=$ Control group.

\begin{tabular}{lccccc}
\hline & $\mathrm{ER}$ & $\mathrm{SC}_{\text {mean }}$ & $\mathrm{SC}_{s d}$ & $\mathrm{CT}_{\text {mean }}$ & $\mathrm{CT}_{s d}$ \\
\hline Feeling of knowing (before) & 0.39 & 1.04 & 0.2 & 1.07 & 0.26 \\
Feeling of closeness (before) & 1.97 & 1.54 & 0.81 & 1.98 & 1 \\
Feeling of closeness (after) & 0.35 & 3.35 & 1.38 & 3.43 & 1.27 \\
Feeling of closeness (after - before) & 0.64 & 1.81 & 1.39 & 1.45 & 1.17 \\
\hline
\end{tabular}

Table 2

Descriptive statistics for HF-HRV (expressed as $m s^{2}$ ) and EMG data (expressed as $\mu V . s$ ) at each time step of the experiment for each experimental condition.

\begin{tabular}{|c|c|c|c|c|c|}
\hline & Measure & $\mathrm{t} 1_{\text {mean }(s d)}$ & $\mathrm{t} 2$ mean $(s d)$ & $\mathrm{t} 3_{\text {mean }(s d)}$ & $\mathrm{t} 4_{\text {mean }(s d)}$ \\
\hline \multirow[t]{2}{*}{ Closeness } & HF-HRV & $5.88(0.84)$ & $5.96(0.87)$ & $5.92(0.77)$ & $6.17(0.96)$ \\
\hline & $\mathrm{EMG}_{\text {corrugator sup }}$ & $9.78(3.78)$ & $8.98(2.76)$ & $8.56(2.41)$ & $8.13(2.43)$ \\
\hline \multirow[t]{2}{*}{ Sham } & HF-HRV & $6.15(0.93)$ & $6.37(0.73)$ & $6.24(0.83)$ & $6.36(0.98)$ \\
\hline & $\mathrm{EMG}_{\text {corrugator supercilii }}$ & $10.26(5.18)$ & $9.17(3.82)$ & $9(3.62)$ & $8.43(3.47)$ \\
\hline
\end{tabular}

Table 3

Comparison of alternative models to the intercept model for HF-HRV and EMG data at each time step of the experiment. Reported values are the ER of the alternative model against the intercept model. $G=G r o u p, R=$ resting-state baseline (at $t 1$ measurement).

\begin{tabular}{lccccc}
\hline & Factor & $\mathrm{t} 1$ & $\mathrm{t} 2$ & $\mathrm{t} 3$ & $\mathrm{t} 4$ \\
\hline HF-HRV & $G$ & 0.70 & 2.79 & 1.19 & 0.46 \\
& $G * R$ & - & 0.70 & 0.49 & 0.34 \\
EMG $_{\text {corrugator supercilii }}$ & $G$ & 0.36 & 0.34 & 0.39 & 0.36 \\
& $G * R$ & - & 0.36 & 0.44 & 0.38 \\
\hline
\end{tabular}

Table 4

Descriptive statistics for reaction times and differences of reaction times during cooperation and control tasks expressed in milliseconds.

\begin{tabular}{lcccc}
\hline & Measure & Alone $_{\text {mean }(s d)}$ & Competition $_{\text {mean }(s d)}$ & Cooperation $_{\text {mean }(s d)}$ \\
\hline Closeness & $R T$ & $349.93(114.78)$ & $293.04(143.54)$ & $346.21(162.04)$ \\
& Diff & $110.31(116.69)$ & $106.35(193.54)$ & $91.59(167.42)$ \\
Control & $R T$ & $325.99(105.32)$ & $281.4(182.04)$ & $356.8(248.06)$ \\
& Diff & $82.66(111.18)$ & $90.8(229.05)$ & $102.32(268.44)$ \\
\hline
\end{tabular}


bioRxiv preprint doi: https://doi org/10.1101/078006; this version posted September 28, 2016. The copyright holder for this preprint (which was not certified by peer review) is the author/funder, who has granted bioRxiv a license to display the preprint in perpetuity. It is made available under aCC-BY-NC-ND 4.0 International license.

DOUBLE-BLIND PROTOCOL IN PROSOCIALITY INDUCTION

Table 5

Comparison of alternative models to the intercept model for reaction times and differences of reaction times during cooperation and control tasks. Reported values are the ER of the alternative model against the intercept model. *** indicates strong evidence toward the alternative model (H1).

\begin{tabular}{lcc}
\hline & $\mathrm{RT}$ & Difference \\
\hline Experimental group & 0.51 & 0.81 \\
Type of task & $>\mathbf{1 0 0}^{* * *}$ & 0.37 \\
Group*Type & 0.46 & 1 \\
\hline
\end{tabular}




\section{Discussion}

This experiment was designed in order to test the influence of ${ }_{655}^{654}$ the experimental generation of social closeness on the autonomic nervous system activity. In addition to physiological measures, we evaluated the participants' level of cooperation. Data collected on self-reported measures, physiological signals (HF-HRV and EMG), and behavioral measures of prosociality (cooperation) did not bring substantial evidence ${ }_{66}$ toward an effect of group: social closeness versus control. $\mathrm{We}_{662}$ expected an interaction with baseline such as low baseline participant on the variable of interest would benefit more from the treatment. Again, this hypothesis was not well supported ${ }^{66}$ by the data. As a consequence, data do not allow conclud- ${ }^{665}$ ing that a protocol of interpersonal closeness generation $\mathrm{can}^{666}$ impact physiological flexibility as indexed by HF-HRV and ${ }^{66}$ secondary by EMG activity, nor behavioral prosociality as ${ }^{66}$ indexed by cooperation. In double-blind sham-controlled ${ }^{66}$ conditions, short term positive interpersonal interaction by ${ }^{670}$ personal information sharing does not differ from a neutral in- ${ }^{67}$ teraction at physiological, behavioral and self-reported levels. ${ }^{672}$ Following the meta-analysis carried out by Shahrestani et al. ${ }^{673}$ (2015), we set up a protocol in a randomized double-blind ${ }^{674}$ design in order to determine whether or not positive affiliative ${ }^{675}$ social interactions could influence the physiological correlates ${ }^{676}$ of prosociality (HF-HRV and EMG activity of the corrugator ${ }^{67}$ supercilii) and other prosocial skills such as cooperation. $\mathrm{A}^{678}$ Aron et al. (1997) and S. L. Brown et al. (2009) have shown ${ }^{679}$ the potential benefits of social closeness on self-reported mea- ${ }^{680}$ sures, behavioral measures and hormonal correlates (proges- ${ }^{681}$ terone) of prosociality. We could not replicate these results in ${ }^{682}$ a laboratory environment (A. Aron et al. (1997) used a more ${ }^{683}$ ecological environment: in a classroom) and importantly with ${ }^{684}$ the experimenter blind to the treatment condition (S. L. Brown ${ }^{685}$ et al. (2009) do not report that the experimental design was ${ }^{686}$ double blind). Moreover, a large part of the effect obtained ${ }^{687}$ by S. L. Brown et al. (2009) was due to a diminution of progesterone level in the control condition which was not ${ }^{689}$ similar to A. Aron et al. (1997) (while the social closeness ${ }^{690}$ condition was the same as A. Aron et al. (1997)). Overall, our ${ }^{691}$ results do not confirm the prosocial benefits nor the efficiency ${ }^{692}$ of social closeness manipulation.

Our data highlights the importance of experimental settings in ${ }^{694}$ the effects observed after interpersonal interactions. With this ${ }^{695}$ study, we show that it is possible to automatically program ex ${ }^{696}$ perimental manipulation of interpersonal relationships, which, ${ }^{697}$ to our knowledge, has never been done before. This paradigm ${ }^{698}$ was expected to test causal predictions concerning the influ- ${ }^{699}$ ence of social interactions on physiological flexibility (Kok \& ${ }^{700}$ Fredrickson, 2010; Porges, 2007). However, we can observe ${ }^{701}$ that results obtained without these methodological precautions ${ }^{702}$ (S. L. Brown et al., 2009) can not be reproduced here. As a ${ }^{703}$ consequence, this questions whether short-term positive social 704 relationships actually impact physiological flexibility. Data705 from the current study does not support this causal pathway from the social to physiological levels of the polyvagal theory (Porges, 2007). As reported by Shahrestani et al. (2015), very few study attempted to test this causal relationship, and when reporting evidence toward a causal nature of the polyvagal theory, methodological biases (S. L. Brown et al., 2009; Kathi J Kemper \& Shaltout, 2011; Willemen et al., 2008) can lead to question the conclusions. Here we show that the interplay between prosociality and heart-brain interactions proposed in the polyvagal theory (Porges, 2007) cannot be explained by the causal role of prosociality.

Several research directions can be explored in order to further explore this question. Our data do not show evidence toward a causal pathway in the polyvagal theory (Porges, 2007), but it worth to decline this kind of paradigm in order to test important modalities influencing social closeness.Indeed, the polyvagal theory suggest an association between sociability and autonomic flexibility allowed by efficient heart-brain interactions. In order to test the causal direction of this theory, it is needed to examine whether manipulating sociability does influence heart-brain interactions. Our study suggests that short term double-blind sham-controlled conditions run against this claim. We propose to test longer social closeness generation protocols in future experiments in order to test the importance of interaction time to influence physiological flexibility. Indeed, it is possible that the amount of retroactions between heart-brain functioning and inter-individual experiences has to be more frequent to be inserted in - even slightly - maintained interactions (Balliet \& Van Lange, 2013; Boyer, Firat, \& Leeuwen, 2015; Keltner, Kogan, Piff, \& Saturn, 2014; Porges, 2007; S. C. Walker \& McGlone, 2013). As a consequence, longer times or several repetitions of social interactions might be necessary to observe a physiological effect at the level of HF-HRV. If carried-out in rigorous methodological conditions, such a protocol could give further insight about the nature of prosocial processes involved in the polyvagal theory (Porges, 2007).

The proposition of Kok \& Fredrickson (2010) concerning the bidirectional interplay between autonomic flexibility and prosociality can corroborate the idea that long-term laboratory manipulation might help to test the polyvagal theory (Porges, 2007). Indeed, the learning mechanisms involved in the management of unsafety and uncertainty in the environment (Brosschot et al., 2016b, 2016a) are likely to be inherently dependent on a minimum of interaction time with strangers in the social domain. For now, the polyvagal theory is not supported by our data in the social to physiological direction. Because we show the feasibility of rigorous methodological set-up, we suggest that further researchers aiming at testing the theory should carry-out experiments based on double-blind protocols.

Conclusions. We aimed to test a possible causal pathway of the polyvagal theory (Porges, 2007) according to which 
prosocial behaviors can positively impact heart-brain dynam-752 ics. Our data does not support the theory in this direction. We suggest that further studies attempting to test the theory $y^{753}$ should focus on rigorous methodological features such as ${ }^{754}$ double-blind design protocols.

Aknowledgements. We thank Stefan Agrigoroaei for tech-757 nical support in data collection. We also thank Anthony Lane,758 Anne Kever, Julie Terache, Bertrand Beffara, Anne Weisger-759 ber, Anael Le Runigo, and Emmanuel Daveau for their work760 on the translation of the social closeness generation procedure. We thank fabrice Damon and Ladislas Nalborczyk for their ${ }^{761}$ useful comments and constructive remarks. This research was ${ }^{762}$ funded by the French CNRS and the Belgian FNRS.

\section{References}

Allaire, J. J., Cheng, J., Xie, Y., McPherson, J., Chang,769 W., Allen, J., .. Hyndman, R. (2016). rmarkdown: Dynamic Documents for R. Retrieved from ${ }^{770}$ https://cran.r-project.org/package $=$ rmarkdown $\quad{ }^{771}$

Aron, A., \& Fraley, B. (1999). Relationship Closeness as In-773 cluding Other in the Self: Cognitive Underpinnings774 and Measures. Social Cognition, 17(2), 140-160775 doi:10.1521/soco.1999.17.2.140

Aron, A., Melinat, E., Aron, E. N., Vallone, R. D., \&777 Bator, R. J. (1997). The Experimental Gener-78 ation of Interpersonal Closeness: A Procedure 779 and Some Preliminary Findings. Personalityr80 and Social Psychology Bulletin, 23(4), 363-377781 doi: $10.1177 / 0146167297234003$

Aust, F., \& Barth, M. (2015). papaja: Create APA manuscripts78s with RMarkdown. Retrieved from https://github 784 $\mathrm{com} / \mathrm{crsh} /$ papaja

Balliet, D., \& Van Lange, P. A. M. (2013). Trust, conflict, and cooperation: A meta-analysis. Psychological 787 Bulletin, 139(5), 1090-1112. doi:10.1037/a0030939788

Bates, D., Maechler, M., Bolker, B., Walker, S., Chris ${ }_{790}^{789}$ tensen, R. H. B., Singman, H., \& Dai, B ${ }_{791}$ (2014). Linear mixed-effects models using Eigen and S4, Package 'Ime4' (Version 1.1-7). Re-792 trieved from http://mirrors.dotsrc.org/pub/pub/cran/793 web/packages/lme4/lme4.pdf

794

Beffara, B., Bret, A. G., Vermeulen, N., \& Mermil ${ }_{790}^{795}$ lod, M. (2016). Resting high frequency heart ${ }_{797}$ rate variability selectively predicts cooperative behavior. Physiology $\mathcal{E}$ Behavior, 164, 417-428798 doi:10.1016/j.physbeh.2016.06.011

Bershad, A. K., Seiden, J. A., \& Wit, H. de. (2016). Effects of buprenorphine on responses to social stimuli in healthy adults. Psychoneuroendocrinology, 63, 439. doi:10.1016/j.psyneuen.2015.09.011

Bertels, J., Deliens, G., Peigneux, P., \& Destrebecqz, A. (2014). The Brussels Mood Inductive Audio Stories (MIAS) database. Behavior Research Methods, 1098-1107. doi:10.3758/s13428-014-0445-3

Bornemann, B., Kok, B. E., Böckler, A., \& Singer, T. (2016). Helping from the heart: Voluntary upregulation of heart rate variability predicts altruistic behavior. Biological Psychology, 119, 54-63. doi:10.1016/j.biopsycho.2016.07.004

Boyer, P., Firat, R., \& Leeuwen, F. van. (2015). Safety, Threat, and Stress in Intergroup Relations: A Coalitional Index Model. Perspectives on Psychological Science, 10(4), 434-450. doi:10.1177/1745691615583133

Briancon, S., Bonsergent, E., Agrinier, N., Tessier, S., Legrand, K., Lecomte, E., ... (ptg), P. T. G. (2010). PRALIMAP: study protocol for a high school-based, factorial cluster randomised interventional trial of three overweight and obesity prevention strategies. Trials, 11(1), 119. doi:10.1186/1745-6215-11-119

Brooks, K. A., Carter, J. G., Dawes, J. J., A Brooks, K., Brooks, K. A., Carter, J. G., ... Dawes, J. J. (2013). A Comparison of VO2 Measurement Obtained by a Physiological Monitoring Device and the Cosmed Quark CPET. Journal Of Novel Physiotherapies, 3(3), 1-2. doi:10.4172/2165-7025.1000126

Brosschot, J. F., \& Thayer, J. F. (1998). Anger inhibition, cardiovascular recovery, and vagal function: A model of the link between hostility and cardiovascular disease. Annals of Behavioral Medicine, 20(4), 326332. doi:10.1007/BF02886382

Brosschot, J. F., Verkuil, B., \& Thayer, J. F. (2016a). Exposed to events that never happen: Generalized unsafety, the default stress response, and prolonged autonomic activity. Neuroscience $\mathcal{E}$ Biobehavioral Reviews. doi:10.1016/j.neubiorev.2016.07.019

Brosschot, J. F., Verkuil, B., \& Thayer, J. F. (2016b). The Default Response to Uncertainty and the Importance of Perceived Safety in Anxiety and Stress: An Evolution-theoretical Perspective. Journal of Anxiety Disorders, 41, 22-34. doi:10.1016/j.janxdis.2016.04.012

Brown, S. L., \& Brown, R. M. (2015). Connecting proso- 
cial behavior to improved physical health: Contri-846 butions from the neurobiology of parenting. $\mathrm{NeU}_{-847}$ roscience and Biobehavioral Reviews, 55, 1-17. doi:10.1016/j.neubiorev.2015.04.004

Brown, S. L., Fredrickson, B. L., Wirth, M. M., Poulin, Ms50 J., Meier, E. A., Heaphy, E. D., ... Schultheiss,, 51 O. C. (2009). Social closeness increases salivary 852 progesterone in humans. Hormones and Behavior,, 53 56(1), 108-111. doi:10.1016/j.yhbeh.2009.03.022 854

Burnham, K. P., \& Anderson, R. (2004). Multimodel In-855 ference: Understanding AIC and BIC in Model Se-856 lection. Sociological Methods $\mathcal{E}$ Research, 33(2), ${ }_{557}$ 261-304. doi:10.1177/0049124104268644

Burnham, K. P., Anderson, D. R., \& Huyvaert, K. P. (2011) $)_{859}$ AIC model selection and multimodel inference in 860 behavioral ecology: Some background, observations,, 861 and comparisons. Behavioral Ecology and Sociobiol - $_{82}$ ogy, 65(1), 23-35. doi:10.1007/s00265-010-1029-6863

Butler, E. a, Wilhelm, F. H., \& Gross, J. J. (2006). Respiratory ${ }^{864}$ sinus arrhythmia, emotion, and emotion regulation ${ }_{865}$ during social interaction. Psychophysiology, 43(6), 666 612-22. doi:10.1111/j.1469-8986.2006.00467.x ${ }_{867}$

Cânovas, M., Domingues, A., \& Sanches, J. M. (2011). Real ${ }^{868}$ Time HRV with smartphone System architecture. In $_{869}$ RecPad (pp. 126-127).

Chambers, J. M. (1992). Linear Models. In J. M. C. (edi ${ }_{872}^{871}$ tors) \& T. J. Hastie (Eds.), Statistical models in s. Wadsworth \& Brooks/Cole, Pacific Grove, Califor-873 nia.

Cheng, X., Li, X., \& Hu, Y. (2015). Synchronous brain ${ }^{875}$ activity during cooperative exchange depends on ${ }^{876}$ gender of partner: A fNIRS-based hyperscanning ${ }_{877}$ study. Human Brain Mapping, 36(6), 2039-2048 878 doi:10.1002/hbm.22754

Costa, V. D., Bradley, M. M., \& Lang, P. J. (2015) From threat to safety: Instructed reversal of defensive reactions. Psychophysiology, 52(3), 325-332 882 doi:10.1111/psyp.12359

Craig, C. L., Marshall, A. L., Sjöström, M., Bauman, $\mathrm{A}_{885}^{884}$ E., Booth, M. L., Ainsworth, B. E., ... Oja, $\mathrm{P}_{886}^{885}$ (2003). International physical activity questionnaire: 12 -country reliability and validity. Medicine and ${ }_{887}$ Science in Sports and Exercise, 35(8), 1381-95 888 doi:10.1249/01.MSS.0000078924.61453.FB 889

Cui, X., Bryant, D. M., \& Reiss, A. L. (2012). NIRSbased hyperscanning reveals increased interper-891 sonal coherence in superior frontal cortex dur-892 ing cooperation. NeuroImage, 59(3), 2430-2437. doi:10.1016/j.neuroimage.2011.09.003

Davies, C. D., Niles, A. N., Pittig, A., Arch, J. J., \& Craske, M. G. (2015). Physiological and behavioral indices of emotion dysregulation as predictors of outcome from cognitive behavioral therapy and acceptance and commitment therapy for anxiety. Journal of Behavior Therapy and Experimental Psychiatry, 46, 35-43. doi:10.1016/j.jbtep.2014.08.002

Denver, J. W., Reed, S. F., \& Porges, S. W. (2007). Methodological issues in the quantification of respiratory sinus arrhythmia. Biological Psychology, 74(2), 286294. doi:10.1016/j.biopsycho.2005.09.005

D’Antono, B., Moskowitz, D. S., Miners, C., \& Archambault, J. (2005). Gender and Communal Trait Differences in the Relations Among Social Behaviour, Affect Arousal, and Cardiac Autonomic Control. Journal of Behavioral Medicine, 28(3), 267-279. doi:10.1007/s10865-005-4663-0

Fridlund, A. J., \& Cacioppo, J. T. (1986). Guidelines for Human Electromyographic Research. Psychophysiology, 23(5), 567-589. doi:10.1111/j.14698986.1986.tb00676.x

Friedman, B. H., \& Thayer, J. F. (1998). Anxiety and autonomic flexibility: a cardiovascular approach. Biological Psychology, 47(3), 243-263. doi:10.1016/S0301-0511(97)00027-6

García, C. A., Otero, A., Vila, X., \& Márquez, D. G. (2013). A new algorithm for wavelet-based heart rate variability analysis. Biomedical Signal Processing and Control, 8(6), 542-550. doi:10.1016/j.bspc.2013.05.006

Grossman, P., \& Taylor, E. W. (2007). Toward understanding respiratory sinus arrhythmia: Relations to cardiac vagal tone, evolution and biobehavioral functions. Biological Psychology, 74(2), 263-285. doi:10.1016/j.biopsycho.2005.11.014

Hagströmer, M., Oja, P., \& Sjöström, M. (2006). The International Physical Activity Questionnaire (IPAQ): a study of concurrent and construct validity. Public Health Nutrition, 9(06), 1127-1132. doi:10.1079/PHN2005898

Heathers, J. A. J. (2014). Everything Hertz: Methodological issues in short-term frequency-domain HRV. Frontiers in Physiology, 5 MAY(May), 177. doi:10.3389/fphys.2014.00177

Heathers, J. A. J., Brown, N. J. L., Coyne, J. C., \& Friedman, H. L. (2015). The Elusory Upward Spiral: A Re- 
DOUBLE-BLIND PROTOCOL IN PROSOCIALITY INDUCTION

analysis of Kok et al. (2013). Psychological Science, 940 26(7), 1140-1143. doi:10.1177/0956797615572908941

Hegyi, G., \& Garamszegi, L. Z. (2011). Using information ${ }_{942}$ theory as a substitute for stepwise regression in ecol-943 ogy and behavior. Behavioral Ecology and Sociobi-944 ology, 65(1), 69-76. doi:10.1007/s00265-010-1036-945 7

Hewig, J., Hagemann, D., Seifert, J., Gollwitzer, M., Nau-947 mann, E., \& Bartussek, D. (2005). Brief Re-948 port. Cognition E Emotion, 19(7), 1095-1109949 doi:10.1080/02699930541000084

Iorfino, F., Alvares, G. A., Guastella, A. J., \& Quintana, D. S. (2016). Cold face test-induced increases in heart rate952 variability are abolished by engagement in a socials53 cognition task. Journal of Psychophysiology, 30(1),954 38-46. doi:10.1027/0269-8803/a000152

Johnstone, J. A., Ford, P. A., Hughes, G., Watson, T., $\&_{957}^{956}$ Garrett, A. T. (2012a). Bioharness( $\left.{ }^{\mathrm{TM}}\right)$ multivariable monitoring device: part. I: validity. Journal of $f_{55}$ Sports Science $\mathcal{E}$ Medicine, 11(3), 400-8.

Johnstone, J. A., Ford, P. A., Hughes, G., Watson, T., $\&_{961}^{960}$ Garrett, A. T. (2012b). Bioharness(TM) Multivariable ${ }_{962}$ Monitoring Device: Part. II: Reliability. Journal of Sports Science $\mathcal{E}$ Medicine, 11(3), 409-17.

Johnstone, J. A., Ford, P. A., Hughes, G., Watson, T., Mitchell ${ }_{965}^{964}$ A. C. S., \& Garrett, A. T. (2012). Field based reli ${ }_{966}^{965}$ ability and validity of the bioharness ${ }^{\mathrm{TM}}$ multivariable monitoring device. Journal of Sports Science $\mathcal{E}_{967}$ Medicine, 11(4), 643-52.

Kass, R., \& Raftery, A. (1995). Bayes Factors. Journal of the American Statistical Association, 90(430), 773-795 ${ }_{971}^{970}$ doi:10.1080/01621459.1995.10476572

Keltner, D., Kogan, A., Piff, P. K., \& Saturn, S. R. (2014) ${ }_{973}^{972}$ The sociocultural appraisals, values, and emotions ${ }_{974}$ (SAVE) framework of prosociality: core processes ${ }_{975}$ from gene to meme. Annual Review of Psychology ${ }_{976}$ 65, 425-60. doi:10.1146/annurev-psych-010213115054

Kemper, K. J., \& Shaltout, H. a. (2011). Non-verbal communication of compassion: measuring psychophysio- ${ }^{979}$ logic effects. BMC Complementary and Alternative Medicine, 11(1), 132. doi:10.1186/1472-6882-11-981 132

Kemper, K. J., Hamilton, C., \& Atkinson, M. (2007) ${ }_{984}^{983}$ Heart rate variability: Impact of differences in ${ }_{985}$ outlier identification and management strategies on common measures in three clinical popu- lations. Pediatric Research, 62(3), 337-342. doi:10.1203/PDR.0b013e318123fbcc

Kobayashi, H. (2009). Does paced breathing improve the reproducibility of heart rate variability measurements? Journal of Physiological Anthropology, 28(5), 225230. doi:10.2114/jpa2.28.225

Kogan, A., Oveis, C., Carr, E. W., Gruber, J., Mauss, I. B., Shallcross, A., ... Keltner, D. (2014). Vagal activity is quadratically related to prosocial traits, prosocial emotions, and observer perceptions of prosociality. Journal of Personality and Social Psychology, 107(6), 1051-63. doi:10.1037/a0037509

Kok, B. E., \& Fredrickson, B. L. (2010). Upward spirals of the heart: Autonomic flexibility, as indexed by vagal tone, reciprocally and prospectively predicts positive emotions and social connectedness. Biological Psychology, 85(3), 432-436. doi:10.1016/j.biopsycho.2010.09.005

Kok, B. E., \& Fredrickson, B. L. (2015). Evidence for the Upward Spiral Stands Steady: A Response to Heathers, Brown, Coyne, and Friedman (2015). Psychological Science, 26(7), 1144-1146. doi:10.1177/0956797615584304

Kowalewski, M. A., \& Urban, M. (2004). Short-and long-term reproducibility of autonomic measures in supine and standing positions. Clinical Science, 106(1), 61-66. doi:10.1042/CS20030119

Larsen, J. T., Norris, C. J., \& Cacioppo, J. T. (2003). Effects of positive and negative affect on electromyographic activity over zygomaticus major and .... Psychophysiology, 40(5), 776-785. doi:10.1111/14698986.00078

Larsen, P. D., Tzeng, Y. C., Sin, P. Y. W., \& Galletly, D. C. (2010). Respiratory sinus arrhythmia in conscious humans during spontaneous respiration. Respiratory Physiology and Neurobiology, 174(1-2), 111-118. doi:10.1016/j.resp.2010.04.021

Lefcheck, J. S. (2016). piecewiseSEM : Piecewise structural equation modelling in $\mathrm{R}$ for ecology, evolution, and systematics. Methods in Ecology and Evolution, 7(5), 573-579. doi:10.1111/2041-210X.12512

Lovibond, P. F., \& Lovibond, S. H. (1995). The structure of negative emotional states: Comparison of the depression anxiety stress scales (DASS) with the Beck Depression and Anxiety Inventories. Behaviour Research and Therapy, 33(3), 335-343. 
bioRxiv preprint doi: https://doi.org/10.1101/078006; this version posted September 28, 2016. The copyright holder for this preprint (which was not certified by peer review) is the author/funder, who has granted bioRxiv a license to display the preprint in perpetuity. It is made available under aCC-BY-NC-ND 4.0 International license.

BRICE BEFFARA ${ }^{1,2,3}$, MARTIAL MERMILLOD ${ }^{1,2}$, NICOLAS VERMEULEN ${ }^{3,4}$

doi:10.1037/1040-3590.10.2.176

1033

Lumma, A. L., Kok, B. E., \& Singer, T. (2015). Is meditaro34 tion always relaxing? Investigating heart rate, heartoss rate variability, experienced effort and likeabilityos6 during training of three types of meditation. Inter ${ }^{0037}$ national Journal of Psychophysiology, 97(1), 38-45. doi:10.1016/j.ijpsycho.2015.04.017

Miller, I. W., Keitner, G. I., Ryan, C. E., Solomon, D. A.1940 Cardemil, E. V., \& Beevers, C. G. (2005). Treatmento41 Matching in the Posthospital Care of Depressed Paro42 tients. American Journal of Psychiatry, 162(11), 1943 2131-2138. doi:10.1176/appi.ajp.162.11.2131 1044

Miller, I. W., Keitner, G. I., Ryan, C. E., Uebelackeriø45 L. A., Johnson, S. L., \& Solomon, D. A1046 (2008). Family treatment for bipolar disorderio47 family impairment by treatment interactions. The 048 Journal of Clinical Psychiatry, 69(5), 732-40. doi:10.1126/scisignal.2001449.Engineering

Miller, J. G., Kahle, S., \& Hastings, P. D. (2015) Roots and Benefits of Costly Giving: Childrenos2 Who Are More Altruistic Have Greater Autoros3 nomic Flexibility and Less Family Wealth. Psychological Science, 26(7), 0956797615578476.054 doi: $10.1177 / 0956797615578476$

Muhtadie, L., Koslov, K., Akinola, M., \& Mendes, Wio57 B. (2015). Vagal flexibility: A physiological predictor of social sensitivity. Journal of Per-1058 sonality and Social Psychology, 109(1), 106-120.059 doi:10.1037/pspp0000016

Piferi, R. L., Kline, K. A., Younger, J., \& Lawler, K. A. (2000)(062 An alternative approach for achieving cardiovascular baseline: Viewing an aquatic video. Interna ${ }^{1063}$ tional Journal of Psychophysiology, 37(2), 207-217.064 doi:10.1016/S0167-8760(00)00102-1 1065

Pinna, G. D., Maestri, R., Torunski, A., Danilowicz+o67 Szymanowicz, L., Szwoch, M., La Rovere, M. T., \&068 Raczak, G. (2007). Heart rate variability measuresio6s a fresh look at reliability. Clinical Science, 113(3), 131-40. doi:10.1042/CS20070055

Porges, S. W. (2007). The polyvagal perspective 072 Biological Psychology, 74(2), 116-143 1073 doi:10.1016/j.biopsycho.2006.06.009

Prinsloo, G. E., Rauch, H. G. L., Lambert, M. I., Muenchip75 F., Noakes, T. D., \& Derman, W. E. (2011). The 076 effect of short duration heart rate variability ( $\mathrm{HRV})_{077}$ biofeedback on cognitive performance during laborto ratory induced cognitive stress. Applied Cognitive
Psychology, 25(5), 792-801. doi:10.1002/acp.1750

Quintana, D. S., \& Heathers, J. A. J. (2014). Considerations in the assessment of heart rate variability in biobehavioral research. Frontiers in Psychology, 5(JUL), 1-10. doi:10.3389/fpsyg.2014.00805

Quintana, D. S., Guastella, A. J., Outhred, T., Hickie, I. B., \& Kemp, A. H. (2012). Heart rate variability is associated with emotion recognition: Direct evidence for a relationship between the autonomic nervous system and social cognition. International Journal of Psychophysiology, 86(2), 168-172. doi:10.1016/j.ijpsycho.2012.08.012

R Core Development Team. (2015). R: a language and environment for statistical computing, 3.2.1. Vienna, Austria: R Foundation for Statistical Computing. doi:10.1017/CBO9781107415324.004

Ramasawmy, S., \& Gilles, P. Y. (2012). The internal and external validities of the Depression Anxiety Stress Scales (DASS-21). International Journal of Psychology, 47(sup1), 1-41. doi:10.1080/00207594.2012.709085

Raposa, E. B., Laws, H. B., \& Ansell, E. B. (2015). Prosocial Behavior Mitigates the Negative Effects of Stress in Everyday Life. Clinical Psychological Science. doi:10.1177/2167702615611073

Rodríguez-Liñares, L., Méndez, A., Lado, M., Olivieri, D., Vila, X., \& Gómez-Conde, I. (2011). An open source tool for heart rate variability spectral analysis. Computer Methods and Programs in Biomedicine, 103(1), 39-50. doi:10.1016/j.cmpb.2010.05.012

Salanave, B., Vernay, M., Szego, E., Malon, A., Deschamps, V., Hercberg, S., \& Castetbon, K. (2012). Physical activity patterns in the French 18-74-year-old population: French Nutrition and Health Survey (Etude Nationale Nutrition Santé, ENNS) 20062007. Public Health Nutrition, 15(11), 2054-9. doi:10.1017/S1368980012003278

Schaefer, A., Nils, F. F., Sanchez, X., \& Philippot, P. (2010). Assessing the effectiveness of a large database of emotion-eliciting films: A new tool for emotion researchers. Cognition E Emotion, 24(7), 1153-1172. doi: $10.1080 / 02699930903274322$

Shahrestani, S., Stewart, E. M., Quintana, D. S., Hickie, I. B., \& Guastella, A. J. (2015). Heart rate variability during adolescent and adult social interactions: A meta-analysis. Biological Psychology, 105, 43-50. 
doi:10.1016/j.biopsycho.2014.12.012

1126

Snipes, M., \& Taylor, D. C. (2014). Model selection and ${ }_{1128}^{127}$ Akaike Information Criteria: An example from wine ratings and prices. Wine Economics and Policy, 3(1),129 3-9. doi:10.1016/j.wep.2014.03.001

Symonds, M. R. E., \& Moussalli, A. (2011). A brief guide to model selection, multimodel inference and model $_{132}$ averaging in behavioural ecology using Akaike's ${ }_{133}$ information criterion. Behavioral Ecology and Soн34 ciobiology, 65(1), 13-21. doi:10.1007/s00265-010 1037-6

Taborsky, B., \& Oliveira, R. F. (2012). Social com com $_{137}$ petence: An evolutionary approach. Trends $\boldsymbol{s}_{138}$ in Ecology and Evolution, 27(12), 679-688. doi:10.1016/j.tree.2012.09.003

Taylor, E. W., Leite, C. A. C., Sartori, M. R., Wang, T., Abe, A. S., \& Crossley, D. A. (2014). The phylogeny and ontogeny of autonomic control of the heart and cardiorespiratory interactions in vertebrates. The Journal of Experimental Biology, 217(5), 690-703. doi:10.1242/jeb.086199

Thayer, J. F., \& Lane, R. D. (2000). A model of neurovisceral integration in emotion regulation and dysregulation. Journal of Affective Disorders, 61(3), 201-216. doi:10.1016/S0165-0327(00)00338-4

Thayer, J. F., Åhs, F., Fredrikson, M., Sollers, J. J., \& Wager, T. D. (2012). A meta-analysis of heart rate variability and neuroimaging studies: Implications for heart rate variability as a marker of stress and health. Neuroscience and Biobehavioral Reviews, 36(2), 747-756. doi:10.1016/j.neubiorev.2011.11.009

Villarejo, M., Zapirain, B., \& Zorrilla, A. (2013). Algorithms Based on CWT and Classifiers to Control Cardiac Alterations and Stress Using an ECG and a SCR. Sensors, 13(5), 6141-6170. doi:10.3390/s130506141

Walker, S. C., \& McGlone, F. P. (2013). The social brain: Neurobiological basis of affiliative behaviours and psychological well-being. Neuropeptides, 47(6), 379393. doi:10.1016/j.npep.2013.10.008

Wells, R., Outhred, T., Heathers, J. A. J., Quintana, D. S., \& Kemp, A. H. (2012). Matter Over Mind: A Randomised-Controlled Trial of Single-Session Biofeedback Training on Performance Anxiety and Heart Rate Variability in Musicians. PLoS ONE, 7(10), e46597. doi:10.1371/journal.pone.0046597

Willemen, A. M., Goossens, F. A., Koot, H. M., \& Schuengel, C. (2008). Physiological reactivity to stress and parental support: comparison of clinical and nonclinical adolescents. Clinical Psychology $\mathcal{E}$ Psychotherapy, 15(5), 340-351. doi:10.1002/cpp.578

Xie, Y. (2013). Dynamic Documents with R and knitr. In Dynamic documents with $r$ and knitr (p. 188). Chapman; Hall/CRC. doi:10.18637/jss.v056.b02

Yoon, J. H., Shah, R. S., Arnoudse, N. M., \& De La Garza, R. (2014). Remote physiological monitoring of acute cocaine exposure. Journal of Medical Engineering $\mathcal{E}$ Technology, 38(5), 244-250. doi:10.3109/03091902.2014.902513

Zephyr. (2014). Zephyr. Retrieved from https://www. zephyranywhere.com 